\title{
Etude du comportement magnétique d'alliages Fe-3\%Si non-orientés, utilisés dans la conception de machines électriques
}

\author{
F. DUMAS, E. HUG*, M. CLAVEL“* et J.L. ILLE
}

Division Electromécanique, Departement Génie Mécanique, Centre de Recherches de Royallieu, Université de Technologie de Compiegne, BP. 649, 60206 Compiegne cedex, France

-Division Electromécanique et Division Mecanique LG2MS, Departement Génie Mécanique, Centre de Recherches de Royallieu, Universite de Technologie de Compiegne, BP. 649, 60206 Compiègne cedex, France

"Division Mécanique LG2MS, Département Génie Mécanique, Centre de Recherches de Royallieu, Université de Technologie de Compiègne, BP. 649, 60206 Compiègne cedex, France

\begin{abstract}
The prediction of core losses in a rotating electrical machine has been estimated by a numerical finite element method and the use of power losses mesurement on a toroidal magnetic lamination (taking account of anisotropy) under sinusoidal induction. The predicted core losses are $40 \%$ lower than the mesured ones. This excess can be attribued to harmonics due to the rotor rotation and modification of the magnetic properties of the material due to the lamination punching and core assemblage.

Mechanical tests and microstructural observations carried out on non-oriented 3\%Si-Fe magnetic sheets showed that the magnetic domain structure is quickly destroyed under 0 to $5 \%$ deformation. Correspondingly, the dislocation density increases following the experimental law: $\log \left(\rho_{d}\right) \alpha+\beta \varepsilon(\alpha, \beta=$ constants). These results are suggested to be related to the magnetic properties alteration due to the sheet forming.
\end{abstract}

\section{INTRODUCTION}

Le calcul des pertes fer dans les machines électriques tournantes est, dans l'état actuel de nos connaissances, très difficile à réaliser. En effet les pertes d'énergie dues à la variation de l'induction dans les matériaux ferromagnétiques dépendent de phénomènes très complexes :

- les pertes par hystérésis,

- les pertes par courant de Foucault.

Ces pertes sont locales et dépendent de l'état microstructural du matériau. Les modèles élaborés de calculs microscopiques des pertes fer [1] sont difficiles à intégrer au niveau macroscopique dès qu'il s'agit d'une gémétrie complexe (e.g. machine électrique tournante). Pour pallier à cette difficulté les utilisateurs de matériaux ferromagnétiques utilisent une méthode de calcul empirique au moyen de caractéristiques magnétiques (cadre d'Epstein,SST,...).

Notre objectif est de prédéterminer, à partir des caractéristiques magnétiques des alliages $\mathrm{Fe}-3 \% \mathrm{Si}$, les pertes fer dans une machine électrique tournante à l'aide de calculs par la méthode des éléments finis. A partir de ces premiers résultats et grâce à des mesures de pertes sur une machine nous essayerons de mettre en évidence la dégradation du comportement magnétique des tôles due aux différentes étapes de construction de cette machine.

Dans cette optique, l'étude du comportement microscopique de l'alliage Fe-3\%Si nonorienté (NO), soumis à des déformations plastiques imposées, pourra permettre de mieux comprendre les phénomènes mis en jeu au cours du processus de dégradation des propriétés magnétiques. Les mesures ainsi réalisées auront pour objectif de cerner d'une part le comportement purement mécanique du matériau, d'autre part de relier l'évolution de ses caractéristiques magnétiques aux modifications de sa microstructure. 


\section{I.CALCUL DES PERTES FER D'UNE MACHINE SYNCHRONE A L'AIDE DE CARACTERISTIQUES MAGNETIQUES.}

\section{Méthodologie proposée}

La détermination de ces pertes nous a conduits à développer des blocs fonctionnels spécifiques à des stratégies de calcul. Les méthodes semi-empiriques développées pour la détermination des pertes fer sont basées sur le calcul du champ d'induction, par la méthode des eléments finis en chaque point de la machine auquel on associe un niveau de perte donné par la caractéristique magnétique du matériau.

La première méthode considère que le champ d'induction reste sinusoïdal dans la machine, même lors de la saturation (calcul en sinusoïdal Equivalent). Ce champ d'induction est décomposé en un champ tournant circulaire et un champ pulsant. Les pertes sont donc déterminées à partir des valeurs crêtes de ces deux champs.

La deuxième considère que le champ d'induction n'est plus sinusoïdal à priori. On fait donc un calcul pas à pas donnant la répartition spatio-temporelle du champ. Par une décomposition de ce champ suivant deux axes principaux nous obtenons deux composantes que nous ramenons à une forme trapézoïdale. Pour chaque élément de la machine, on identifie la valeur des pertes fer, correspondant à la valeur crête du trapèze.

La dégradation des caractéristiques magnétiques de la tôle, consécutive aux différentes étapes de la construction d'une machine, est appréciée par l'évolution du niveau des pertes fer mesurees sur un banc d'essais.

\section{Résultats_numériques}

2.1Cartographie spatio-temporelle du champ dans la machine

Les mesures et les calculs sont réalisés sur une machine synchrone de $2 \mathrm{~kW}, 8$ pôles, $50 \mathrm{~Hz}, 750 \mathrm{tr} / \mathrm{mn}$. Pour les mesures de pertes son rotor est entraîné à la vitesse de synchronisme par une autre machine synchrone.

Il nous est paru intéressant de montrer que la part du champ tournant par rapport au champ pulsant est relativement peu importante dans cette machine. Si les calculs font apparaitre clairement qu'il existe un champ tournant important dans certaines parties (figl) il est néanmoins nécessaire de montrer que ce champ est trés localisé et que sur la totalité du volume du matériau magnétique de la machine sa part relative n'excède pas $11 \%$ du champ total .

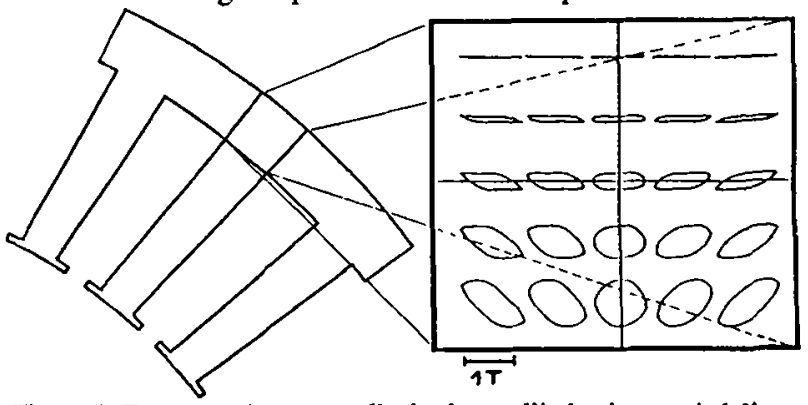

Il est bien entendu que cette proportion de champ tournant n'est pas égale à la part des pertes due à ce champ tournant qui est bien supérieure à $11 \%$ des pertes totales.

Eigure 1: Forme spatio-temporelle du champ d'induction au pied d'une dent (calcul EFEL).

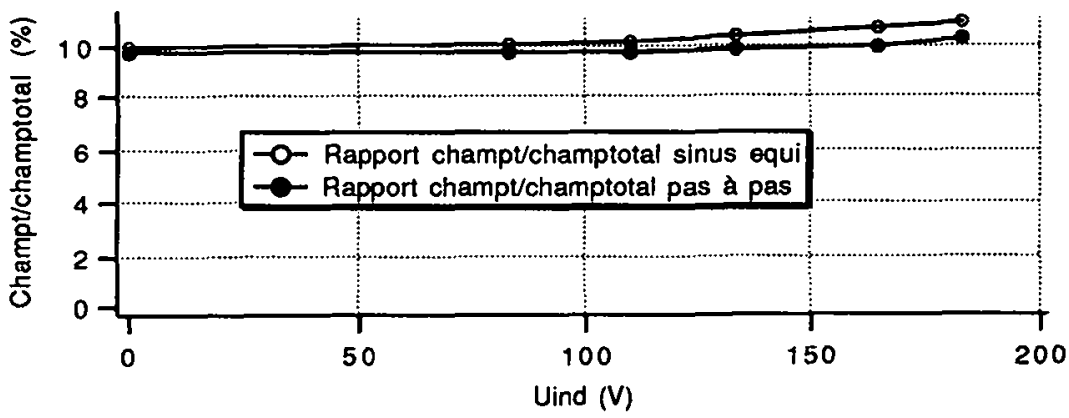

Eigure 2: Evolution du rapport champ tournant / champ total en fonction de la saturation (calcul EFEL). 
Les courbes précédentes (fig2) montrent l'effet de la saturation sur la proportion de champ tournant dans la machine.

\subsection{Adéquation calcul-expérimentation}

Par les deux méthodes explicitées plus haut, nous avons calculé les pertes fer dans le stator de la machine. Les calculs obtenus (fig3) montrent l'importance de la caractéristique $P(B)$ du matériau. En effet, pour la même tôle deux caractéristiques de pertes ont été élaborées. Une sur le cadre d'Epstein, l'autre sur un tore ce qui permet de tenir compte de l'anisotropie du matériau. Les courbes obtenues laissent voir que le calcul sous-estime les pertes fer (40\%). Cet écart peut s'expliquer par l'effet de la réluctance variable des dents qui introduit une variation du champ lors de la rotation du rotor $(300 \mathrm{~Hz}$ au synchronisme) et par la dégradation des caractéristiques magnétiques des tôles lors de leur poinçonnage et assemblage.

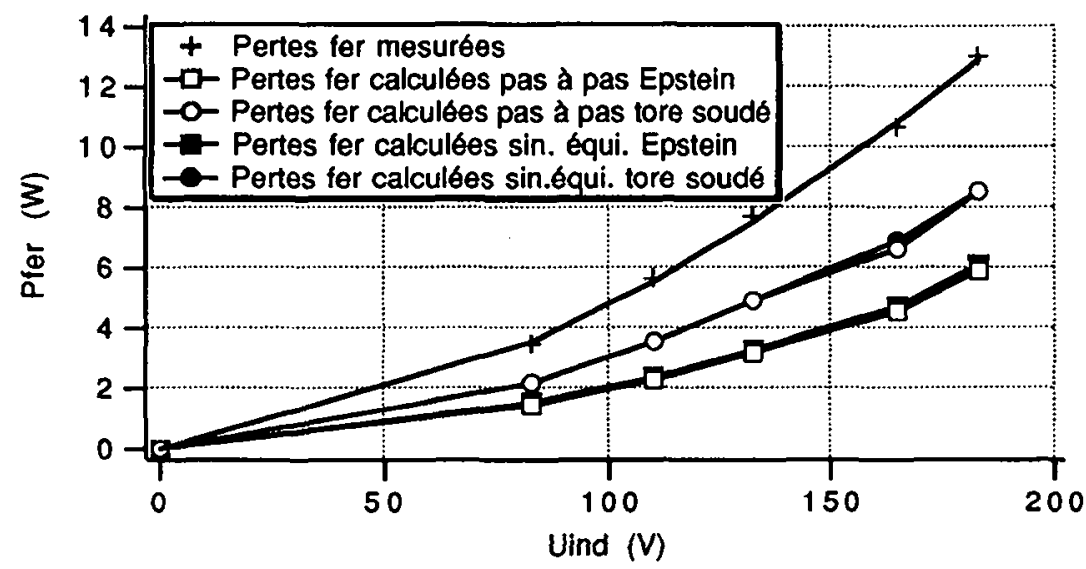

Eigure 3: Pertes fer stator (calcul EFEL).

\section{Perspectives}

Notre travail actuel consiste à développer un code de calcul tenant compte de l'effet de réluctance variable d'entrefer due à la rotation du rotor dans la machine [2] afín de déterminer les pertes avec une meilleure précision qu'à l'heure actuelle.

\section{II.INFLUENCE DE LA MISE EN OEUVRE SUR LES PROPRIETES MAGNETIQUES D'ALLIAGES Fe-3\%Si.}

\section{Techniques expérimentales}

Des essais de traction à déformation plastique imposée ont été effectués à température ambiante sur des tôles magnétiques Fe-3\%Si NO. Les éprouvettes de traction ont été découpées selon plusieurs orientations par rapport à la direction de laminage. réalisé:

D'un point de vue microscopique, pour chaque pourcentage de deformation, nous avons

-un examen en microscopie optique de la structure générale du matériau,

-une visualisation des domaines magnétiques par des méthodes colloïdales appropriées [3], [4],

-des observations en Microscopie Electronique à Transmission (MET) de la configuration des dislocations. 
Au niveau macroscopique, la réalisation d'un banc de mesures (tracé de la courbe d'aimantation et du cycle d'hystérésis), nous permet l'enregistrement des caractéristiques magnétiques pour chaque éprouvette déformée par traction. Pour l'instant, les résultats obtenus sont relatifs (comparaison des courbes entre éprouvette non-déformée et éprouvette déformée). Afin de donner un caractère absolu à nos mesures, nous cherchons actuellement à évaluer par une méthode d'éléments finis le pourcentage de flux de fuites du banc dans le but de préciser la quantité de champ magnétique traversant effectivement l'éprouvette. Enfin, la comparaison, pour le matériau vierge de toute deformation, des mesures obtenues sur notre banc et de celles réalisées sur un cadre Epstein devrait nous permettre de nous affranchir totalement de la géométrie de l'éprouvette.

\section{Résultats obtenus}

Les différentes observations microscopiques réalisées nous ont permis de constater:

- une destruction rapide de la structure en domaines magnétiques. La configuration initiale comporte de nombreux domaines d'orientations anti-parallèles (structure principale de densité volumique $\Omega_{1}$ ) ainsi qu'une proportion relativement importante de pics de fermeture (structure secondaire de densité volumique $\Omega_{2}$ ) d'aimantation inverse aux domaines les contenant, que l'on trouve notamment dans des zones proches de joints de grains en raison de l'orientation aléatoire des grains les uns par rapport aux autres. Pour une déformation plastique $\varepsilon_{\mathrm{p}}=5 \%$, $\Omega_{2}$ augmente sensiblement par rapport à $\Omega_{1}$, et des domaines d'orientation transverse à $D L$ apparaissent dans le matériau (figure 4).
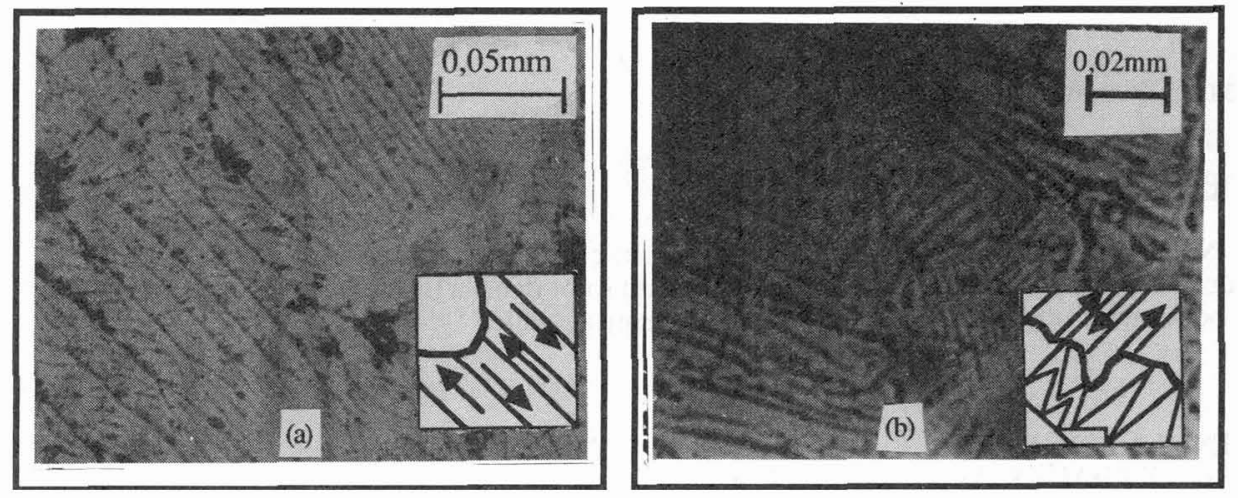

Eigure 4: Destruction de la structure en domaines magnétiques du matériau avec la déformation. (a): $\varepsilon=0,8 \%$ : (b): $\varepsilon=5 \%$

- une augmentation de la densité de dislocations $\rho_{d}$ avec $\varepsilon_{p}$ (figure 5), qui suit une loi du type: $\log \left(\rho_{d}\right)=\alpha+\beta \varepsilon_{p}$, où $\alpha$ et $\beta$ sont des constantes. Cette loi a été obtenue par des techniques de comptage de dislocations sur des clichés photographiques (Analyse par intersection de SmithGuttman, 1953, [5]). De plus la configuration des dislocations, relativement isotrope entre 0 et $10 \%$ de déformation, devient par la suite fortement anisotrope, les défauts ayant tendance à se regrouper en amas. Ceci a déjà été observé précédemment dans du fer de haute pureté [6]. 

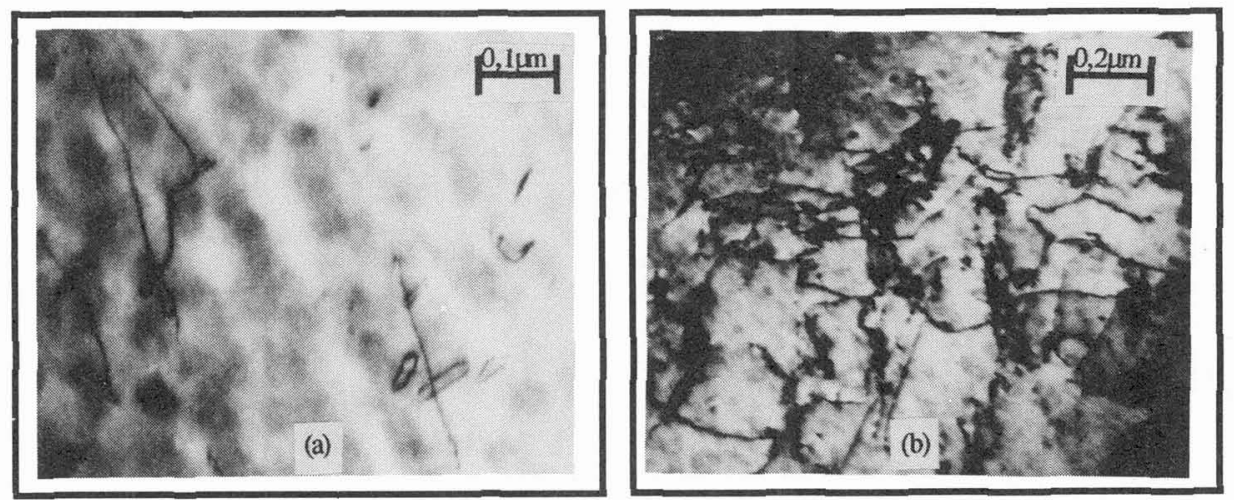

Figure 5: évolution de la densité de dislocations avec le taux de déformation imposé, traction dans la direction de laminage. (a): $\varepsilon=0,8 \%$; (b): $\varepsilon=5 \%$. Dislocations vis de type $\{110\} a / 2<111>$.

\section{CONCLUSION}

Les essais en cours de réalisation nous permettront de relier la dégradation des caractéristiques magnétiques du matériau à sa densité de dislocations, par l'intermédiaire de la déformation plastique imposée. L'analyse des modifications de la structure magnétique avec les déformations devrait nous faire mieux comprendre les mécanismes sous-jacents à ces phénomènes de dégradation. Ces résultats pourront être appliqués sur des exemples simples de mise en oeuvre tels que découpage ou poinçonnage de tôles. L'étape suivante sera d'incorporer ces résultats dans un code de calcul permettant de mieux préteterminer les pertes fer dans les machines électriques tournantes.

\section{BIBLIOGRAPHIE}

[1]. BURAIS N. : "Etude et modélisation des pertes dans les circuits magnétiques en régime non sinusoïdal à fróquence industrielle élevée"; Thèse ; Ecole centrale de LYON,1981.

[2]. FELIACHI M. : "Contribution au calcul du champ electromagnétique par la méthode des éléments finis en vue d'une modélisation dynamique de machines électriques" ; Thèse de Doctorat,CNAM,1981.

[3]. CAREY R. - ISAAC E.D.: "Magnetic domains and techniques for their observation"; English universities press limited, London, 1966.

[4]. GOTO K. - SAKURAI T. - KITAKAMI O.: "Observations on perpendicular magnetic recordings of high recording densities on Co-Cr films by the colloïd-SEM method"; Jap. J. of Appl. Phys., vol.25, n9, 1986

[5]. HIRSCH P.B. - HOWIE A. - NICHOLSON R.B. - PASHLEY D.W. - WHELAN M.J.: "Electron microscopy of thin cristals"; London, Butterworths, 1965.

[6]. DEGAUQUE J. - ASTIE B.: "Evolution of the configuration of magnetic domains interacting with structural defects in high purity iron"; phys. stat. sol. (a), $\mathrm{n}^{\circ 74,1982 .}$ 